\title{
Galectin-3 Enhances Avian H5N1 Influenza A Virus-Induced Pulmonary Inflammation by Promoting NLRP3 Inflammasome Activation
}

\author{
Yu-Jung Chen, ${ }^{*}$ Sheng-Fan Wang, ${ }^{* \dagger}$ I-Chun Weng, ${ }^{*}$ Ming-Hsiang Hong, ${ }^{*}$ Tzu-Han Lo, ${ }^{*}$ Jia-Tsrong Jan,,${ }^{\ddagger}$ Li-Chung Hsu, \\ Huan-Yuan Chen, ${ }^{*}$ and Fu-Tong Liu*
}

\begin{abstract}
From the Institute of Biomedical Sciences, * Academia Sinica, Taipei; the Department of Medical Laboratory Science and Biotechnology, ${ }^{\dagger}$ Kaohsiung Medical University, Kaohsiung; the Genomics Research Center, ${ }^{\ddagger}$ Academia Sinica, Taipei; and the Institute of Molecular Medicine, ${ }^{\S}$ National Taiwan University, Taipei, Taiwan
\end{abstract}

\author{
Accepted for publication \\ December 28, 2017 \\ Address correspondence to \\ Fu-Tong Liu, M.D., Ph.D., or \\ Huan-Yuan Chen, Ph.D., Insti- \\ tute of Biomedical Sciences, \\ Academia Sinica, 128 Sec. 2 , \\ Academia Rd. Nankang, Taipei \\ 115, Taiwan, R.O.C. E-mail: \\ ftliu@ibms.sinica.edu.tw or \\ hchen9@ibms.sinica.edu.tw.
}

\begin{abstract}
Highly pathogenic avian influenza A H5N1 virus causes pneumonia and acute respiratory distress syndrome in humans. Virus-induced excessive inflammatory response contributes to severe disease and high mortality rates. Galectin-3, a $\beta$-galactoside-binding protein widely distributed in immune and epithelial cells, regulates various immune functions and modulates microbial infections. Here, we describe galectin-3 up-regulation in mouse lung tissue after challenges with the H5N1 influenza virus. We investigated the effects of endogenous galectin-3 on $\mathrm{H} 5 \mathrm{~N} 1$ infection and found that survival of galectin-3 knockout (Gal-3KO) mice was comparable with wild-type (WT) mice after infections. Compared with infected WT mice, infected Gal-3KO mice exhibited less inflammation in the lungs and reduced IL-1 $\beta$ levels in bronchoalveolar lavage fluid. In addition, the bone marrow-derived macrophages (BMMs) from Gal-3KO mice exhibited reduced oligomerization of apoptosis-associated speck-like proteins containing caspase-associated recruitment domains and secreted less IL-1 $\beta$ compared with BMMs from WT mice. However, similar levels of the inflammasome component of nucleotide oligomerization domain-like receptor protein 3 (NLRP3) were observed in two genotypes of BMMs. Coimmunoprecipitation data indicated galectin-3 and NLRP3 interaction in BMMs infected with H5N1. An association was also observed between galectin-3 and NLRP3/apoptosis-associated speck-like proteins containing caspase-associated recruitment domain complex. Combined, our results suggest that endogenous galectin-3 enhances the effects of H5N1 infection by promoting host inflammatory responses and regulating IL-1 $\beta$ production by macrophages via interaction with NLRP3. (Am J Pathol 2018, 188: 1031-1042; https://doi.org/10.1016/j.ajpath.2017.12.014)
\end{abstract}

H5N1 is a highly pathogenic avian influenza A virus (IAV) that was initially confined to poultry, but it is now recognized as a human infectious agent associated with a case-fatality rate of approximately $60 \%{ }^{1}$ Clinical features of human $\mathrm{H} 5 \mathrm{~N} 1$ infections include typical influenza-like symptoms during early stages and rapidly progressive viral pneumonia. Complications can include acute respiratory distress syndrome and multiple organ failure. ${ }^{2,3}$ Lung pathologic process and progressive respiratory failure are considered the primary causes of H5N1-associated deaths. ${ }^{4}$ According to previous studies, virus-induced excessive acute inflammatory responses in hosts contribute to H5N1 pathogenesis. ${ }^{1,5}$ Those responses include massive influxes of inflammatory cells into the lungs and aberrant production of cytokines and chemokines, resulting in pulmonary immunopathology and increased tissue injury. ${ }^{5-7}$ A growing body of evidence points to activated macrophages as primary contributors of elevated cytokine

Supported by Academia Sinica and Ministry of Science and Technology, Republic of China grants MOST103-100 (H.-Y.C.), MOST 106-0210-0115-02 (F.-T.L.), and MOST 106-2321-B-037-001 (S.-F.W.). Anti-caspase-1 (p20) was a gift from Genentech.

Disclosures: None declared. 
production and subsequent immunopathology induced by H5N1 infection. ${ }^{8,9}$

IL-1 $\beta$ is a potent proinflammatory cytokine involved in the acute lung immunopathology of IAV infections. ${ }^{10}$ Primarily produced by activated monocytes and macrophages, ${ }^{11,12}$ IL-1 $\beta$ induces the expression of proinflammatory mediators, cytokines, and chemokines that trigger leukocyte production. ${ }^{12}$ Respiratory epithelial cells and macrophages sense the virus during an infection, thereby triggering IL-1 $\beta$ production by the activation of nucleotide oligomerization domain-like receptor protein 3 (NLRP3) signal. ${ }^{13-15}$ This results in the increased recruitment of inflammatory cells such as neutrophils and monocytes to infection sites. Several reports suggest that two signals are required to activate NLRP3 inflammasome (a cytoplasmic multiprotein complex). The first (priming) signal is triggered by a toll-like receptor agonist such as viral genomic RNA, resulting in the transcription of pro-IL-1 $\beta$ and NLRP3 via the NF- $\mathrm{B}$ pathway. ${ }^{16} \mathrm{Sec}-$ ondary stress-related signals include reactive oxygen species, $\mathrm{K}^{+}$efflux, and lysosomal rupture signals that promote NLRP3 inflammasome complex assembly. ${ }^{13,17}$ Activated NLRP3 inflammasomes act as platforms for caspase-1 activation that consequently cleaves pro-IL-1 $\beta$ into mature IL-1 $\beta .^{18}$

Galectins are a family of $\beta$-galactoside-binding animal lectins defined by a conserved carbohydrate-binding domain of approximately 135 amino acids with affinities for $\beta$-galactose-containing glycoconjugates. ${ }^{19}$ Galectin-3 (approximately $30 \mathrm{kDa}$ ) is the only chimera-type galectin that contains a single carbohydrate-binding domain at the Cterminal region, as well as an extended nonlectin $\mathrm{N}$-terminal part that contributes to galectin-3 self-association. ${ }^{20}$ Galectin3 is widely distributed in a variety of tissues and expressed by various immune and epithelial cells. ${ }^{21,22}$ It is primarily detected in the cytoplasm, but it can be translocated to the cell nucleus. It is also secreted into extracellular environments by undefined secretory pathways. ${ }^{23}$ Researchers have described multiple galectin-3 functions in a range of physiological and pathologic processes that involve intracellular and extracellular functions. ${ }^{22,24}$ The importance of galectin-3 in the development and regulation of immune homeostasis and responses to infections has been well demonstrated. ${ }^{24,25} \mathrm{~A}$ number of in vivo studies that used galectin-3-knockout (Gal$3 \mathrm{KO})$ mice have confirmed the proinflammatory effect of endogenous galectin- $3 .{ }^{26}$ For example, Gal-3KO mice have been shown to exhibit attenuated inflammatory response and reduced leukocyte infiltration compared with wild-type (WT) mice in thioglycollate-induced peritoneal inflammation ${ }^{27,28}$ and Streptococcus pneumoniae infections. ${ }^{29}$

Galectin-3 is up-regulated during lung injury, allergic airway inflammation, and airway infections. ${ }^{29-31}$ Therefore, it was hypothesized that a correlation existed between endogenous galectin-3 and H5N1-induced pulmonary immune response. During this study we observed up-regulated galectin-3 in a mouse model challenged with the $\mathrm{H} 5 \mathrm{~N} 1$ virus and more severe airway inflammation and greater IL$1 \beta$ production in WT mice than Gal-3KO mice, suggesting a promoting effect of endogenous galectin-3 on H5N1induced inflammation. With the use of mouse macrophages for in vitro studies, an association was found between galectin-3 and NLRP3-specifically, galectin-3 promoted NLRP3 inflammasome assembly and activation in response to $\mathrm{H} 5 \mathrm{~N} 1$ infections. Our data demonstrate a positive role for galectin-3 in $\mathrm{H} 5 \mathrm{~N} 1$ infection-induced inflammation by NLRP3 inflammasome activation and IL-1 $\beta$ production.

\section{Materials and Methods}

\section{Cell Lines}

Madin-Darby canine kidney cells (ATCC PTA-6500, Manassas, VA) were used for influenza virus propagation. Madin-Darby canine kidney, J774.1 (a mouse macrophage cell line), and HEK293T cells were maintained in Dulbecco's Modified Eagle Medium (Gibco, Grand Island, NY) supplemented with $10 \%$ heat-inactivated fetal bovine serum (Gibco), $10 \mathrm{U} / \mathrm{mL}$ penicillin (Sigma-Aldrich, St. Louis, $\mathrm{MO}$ ), and $100 \mu \mathrm{g} / \mathrm{mL}$ streptomycin sulfate (Gibco) at $37^{\circ} \mathrm{C}$ in a $5 \% \mathrm{CO}_{2}$ humidified incubator.

\section{Virus}

Influenza H5N1 NIBRG-14 is an attenuated reassortant strain that possesses modified HA and NA genes derived from A/Vietnam/1194/2004 (H5N1) and six internal genes from A/Puerto Rico/8/34 (H1N1). ${ }^{32}$ The NIBRG-14 virus was propagated in Madin-Darby canine kidney cells. Virus titers were determined by plaque assays and $50 \%$ tissue culture infective dose assays. All experiments that involved the handling of H5N1 NIBRG-14 viruses in vitro or in vivo were conducted in the BSL-2 ${ }^{+}$laboratory at the Academia Sinica Institute of Biomedical Sciences and Genomics Research Center.

\section{Mouse Infections}

Gal-3KO mice with C57BL/6 backgrounds were generated as previously described, ${ }^{27}$ and WT mice with identical genetic backgrounds were used as controls. All animal procedures and experimental protocols were approved by the Institutional Animal Care and Utilization Committee of Academia Sinica and were performed according to committee guidelines.

Groups of female C57BL/6 WT and Gal-3KO mice (8 to 9 weeks old) were intranasally inoculated with 10 times the half-lethal doses $\left(\mathrm{LD}_{50}\right)$ of H5N1 NIBRG-14 virus $(50 \mu \mathrm{L})$ on day 0 . Body weight, body temperature, and survival data were recorded for 14 days. Sample collection and virus challenges were performed under sodium pentobarbital anesthesia (60 to $80 \mathrm{mg} / \mathrm{kg}$ ); every effort was made to minimize animal 
suffering. Mice showing $>25 \%$ body weight loss were considered as reaching an experimental end point and were humanely euthanized according to guidelines from the Institutional Animal Care and Use Committee.

\section{Preparation of Mouse Bone Marrow-Derived Macrophages}

Both ends of mouse femurs and tibias were cut and flushed with $5 \mathrm{~mL}$ of RPMI 1640 (Invitrogen, Carlsbad, CA) by using a 27-gauge $\times 1 / 2$-inch needle (Terumo, Tokyo, Japan). Bone marrow cells were strained (40- $\mu \mathrm{m}$ mesh) (BD Biosciences, San Jose, CA) and seeded in complete medium that consisted of RPMI 1640 that contained $10 \%$ fetal bovine serum, $1 \times$ nonessential amino acids (Gibco), $20 \mathrm{mmol} / \mathrm{L}$ HEPES (Gibco), and $10 \mathrm{ng} / \mathrm{mL}$ mouse granulocyte-macrophage colony-stimulating factor (PeproTech, Rocky Hill, NJ) for macrophage differentiation. One-half of culture medium was replaced every 2 days with fresh complete medium. Differentiated macrophages were harvested after 7 to 10 days with Cellstripper (Corning Cellgro, Manassas, VA) and used for virus infection experiments.

\section{Cell Culture Infection with H5N1}

Bone marrow-derived macrophages (BMMs) or J774.1 cell monolayers were cultured overnight in 6-well plates, washed two more times with phosphate-buffered saline (PBS), inoculated with H5N1 NIBRG-14 (multiplicity of infection $=1$ ) in influenza virus growth medium, and incubated for 1 hour at $37^{\circ} \mathrm{C}$. Cells were washed two additional times with PBS, and fresh cell culture medium was placed in each well before incubation at $37^{\circ} \mathrm{C}$ in a $5 \%$ $\mathrm{CO}_{2}$ humidified incubator. Cell-free supernatants were collected after 6 and 24 hours for enzyme-linked immunosorbent assay (ELISA) cytokine analyses.

\section{RNA Extraction and RT-PCR}

Mice infected with H5N1 NIBRG-14 virus were sacrificed 1 to 5 days after infection. Lungs were perfused with 10 $\mathrm{mL}$ of PBS injected into right heart ventricles, harvested, and homogenized in $1 \mathrm{~mL}$ of TRIzol (Invitrogen) with a TissueLyser Adapter Set and TissueLyser II (both Qiagen, Valencia, CA). After centrifugation at $12,000 \times g$ for 15 minutes at $4{ }^{\circ} \mathrm{C}$, supernatants were collected and used for total RNA extraction. Extracted RNA quality and quantities were measured with a NanoDrop 2000C spectrophotometer (Thermo Fisher Scientific, Waltham, MA). Next, $1 \mu \mathrm{g}$ of extracted RNA was reverse-transcribed into cDNA by using iScript Reverse Transcription Supermix for quantitative real-time RT-PCR (RT-qPCR) (Bio-Rad, Hercules, CA) according to the manufacturer's instructions.
H5N1 Virus and Cytokine mRNA Quantification by RealTime PCR

To measure H5N1 NIBRG-14 virus levels in lung tissue or cell culture supernatants, quantitative real-time PCR was performed with an ABI 7500 Real-Time PCR machine (Applied Biosystems, Foster City, CA) to amplify cDNA synthesized from extracted RNA with a FastStart Universal Probe Master (Roche Applied Science, Indianapolis, IN). For viral RNA detection, H5HA gene-specific primers (forward: 5'-AGGTCCGACTACAGCTTAGGG-3', reverse: $5^{\prime}$-TGATAGAACTCGAAACAACCGTTA- $3^{\prime}$ ) and probes designed by the Universal Probe Library Assay Design Center (Roche Applied Science) were used for realtime PCR amplification. H5N1 NIBRG-14 quantities were calculated by interpolation from standard curves constructed by the parallel running of 10 -fold serial dilutions of known numbers of plasmids that contained H5HA gene segments.

Inflammasome component and cytokine mRNA levels in mouse lung tissues or BMMs were detected by real-time quantitative PCR using the primers IL-1 $\beta$ (forward: $5^{\prime}$ AGTTGACGGACCCCAAAAG- ${ }^{\prime}$, reverse: $5^{\prime}$-AGCTGGATGCTCTCATCAGG-3'), NLRP3 (forward: 5'-CCCTTGGAGACACAGGACTC- $3^{\prime}$, reverse: $5^{\prime}$-GAGGCTGCAGTTGTCTAATTCC- $3^{\prime}$ ), tumor necrosis factor (TNF)- $\alpha$ (forward: 5'-CAGCCTCTTCTCCTTCCTGAT-3', reverse: 5'-GCCAGAGGGCTGATTAGAGA-3'), and chemokine (C-C motif) ligand 2 (forward: $5^{\prime}$-CATCCACGTGTTGGCTCA-3' ${ }^{\prime}$, reverse: 5'-GATCATCTTGCTGGTGAATGAGT-3').

\section{Histochemical Analysis}

Three days after infection, infected mouse lungs were perfused with PBS and fixed with $1 \mathrm{~mL}$ of $4 \%$ paraformaldehyde (Sigma-Aldrich) by tracheas. Lungs were fixed overnight with $10 \%$ formaldehyde (Merck, Darmstadt, Germany), embedded in paraffin, sectioned, and stained with hematoxylin and eosin. Immunohistochemical staining was performed on paraffin-embedded lung sections. Endogenous peroxidase activity and nonspecific protein binding were blocked by using Hydrogen Peroxide Block (Thermo Fisher Scientific) and Ultra V Block (Thermo Fisher Scientific), respectively. Sections were incubated with rabbit anti- galectin-3 antibodies (1:1000 dilution; made in our laboratory) or normal rabbit IgG antibodies (Abcam, Cambridge, $\mathrm{MA}$ ) as isotype control at working concentration of $0.5 \mu \mathrm{g} /$ $\mathrm{mL}$. Bound antibodies were detected by incubation with Histofine Simple Stain Mouse MAX PO (Nichirei, Tokyo, Japan), followed by 3, 3-diaminobenzidine (Vector, Burlingame, CA) treatment for 5 minutes at room temperature. Slides were counterstained with hematoxylin.

\section{ELISAs}

Mouse lungs were harvested and homogenized in PBS. Bronchoalveolar lavage (BAL) fluid was collected by 
injecting $1 \mathrm{~mL}$ of PBS into the alveolar spaces of infected mice, followed by three gentle washings via tracheas with the use of an 18-gauge $\times 1 \frac{1}{2}$-inch needle (Terumo) on day 3 after infection. Collected BAL fluid and lung homogenates were centrifuged at $14,000 \times g$ for 15 minutes at $4^{\circ} \mathrm{C}$. Cytokine concentrations in the supernatants were estimated by using Ready-Set-Go! ELISA Sets (eBioscience, San Diego, CA). Cytokine levels in cell-free supernatants from virus-infected BMMs were also measured by using ELISA kits. Absorbance at $450 \mathrm{~nm}$ was measured with an Infinite 200 PRO spectrophotometer (Tecan, Research Triangle Park, NC).

\section{Immunoblot Analysis}

Cells were lyzed in RIPA lysis buffer on ice. Total proteins $(20 \mu \mathrm{g})$ were used for SDS-PAGE analyses. Separated proteins were transferred to polyvinylidene fluoride membranes (Millipore, Bedford, MA). Membranes were blocked overnight at $4{ }^{\circ} \mathrm{C}$ for nonspecific protein binding in blocking buffer with $5 \%$ milk (BD Biosciences) in $0.1 \%$ Tween 20 in PBS before incubation with anti-galectin-3, anti-caspase-1 (p20) (gifts from Genentech, San Francisco, CA), anti-IL-1 $\beta$ (R\&D, Minneapolis, MN), anti-NLRP3 (Adipogen, San Diego, CA), anti-apoptosis-associated speck-like proteins containing caspase-associated recruitment domain (ASC; Adipogen), and anti- $\alpha$-tubulin antibodies (Epitomics, Burlingame, CA). Membranes were washed with $0.1 \%$ Tween 20 in PBS to remove unbound primary antibodies and incubated with horseradish peroxidase-conjugated secondary antibodies at room temperature for 1 hour. After another washing with $0.1 \%$ Tween 20 in PBS, membranes were developed with WesternBright ECL (enhanced chemiluminescence) HRP (horseradish peroxidase) Substrate (Advansta, Menlo Park, CA). Hybridized protein bands were visualized with a BioSpectrum Auto Imaging System (UVP, Upland, CA) and quantified with ImageQuant 5.0 software (Molecular Dynamics, Sunnyvale, CA).

\section{NLRP3 Inflammasome Complex Reconstitution}

HEK293T cells were transfected with plasmids encoding pro-IL-1 $\beta \quad(200 \quad \mathrm{ng})$, flag-tagged ASC (50 ng), pro-caspase-1 (30 ng), and NLRP3 (75 ng) according to Effectene Transfection Reagent protocols as described by Qiagen. Proteins in supernatants were precipitated with trichloroacetic acid (Sigma-Aldrich), and mature IL-1 $\beta$ was detected by Western blot analysis.

\section{ASC Pyroptosome Cross-Link Assays}

BMMs were harvested 15 hours after infection and lyzed in buffer that contained $20 \mathrm{mmol} / \mathrm{L}$ HEPES-KOH (pH 7.5), 10 $\mathrm{mmol} / \mathrm{L} \mathrm{KCl}, 1.5 \mathrm{mmol} / \mathrm{L} \mathrm{MgCl} 2,1 \mathrm{mmol} / \mathrm{L}$ EDTA, $1 \mathrm{mmol} /$ L EGTA, $320 \mathrm{mmol} / \mathrm{L}$ sucrose, and protease inhibitor cocktail (Calbiochem, San Diego, CA) (15 passes on ice with the use of a 27-gauge syringe). Lysates were centrifuged at $520 \times g$ for 8 minutes to remove cell nuclei. Supernatants were diluted with 3-[(3-cholamidopropyl)dimethylammonio]-1-propanesulfonate (CHAPS) buffer (20 mmol/L HEPES-KOH, pH 7.5, $5 \mathrm{mmol} / \mathrm{L}$ $\mathrm{MgCl}_{2}, 0.5 \mathrm{mmol} / \mathrm{L}$ EGTA, $0.1 \%$ CHAPS) plus $0.1 \mathrm{mmol} / \mathrm{L}$ fresh phenylmethane sulfonyl fluoride and protease inhibitor cocktail, and they were centrifuged at $4000 \times g$ for 8 minutes. Pellets were resuspended in CHAPS buffer with $2 \mathrm{mmol} / \mathrm{L}$ disuccinimidyl suberate cross-linker (Thermo Fisher Scientific) and held at room temperature for 30 minutes. After centrifugation at $4000 \times g$ for 10 minutes, pellets were resuspended with $1 \times$ IAV sample buffer and fractionated on $12 \%$ SDS polyacrylamide gels, followed by immunoblotting with anti-ASC antibodies.

\section{Immunofluorescence}

293T cells were seeded on poly-L-lysine-coated glass coverslips (Sigma-Aldrich) and transfected with plasmids that encoded galectin-3, NLRP3, and ASC for 48 hours. Cells were fixed with $4 \%$ paraformaldehyde and were permeabilized with $0.05 \%$ saponin (Sigma-Aldrich). After blocking with buffer, cells were stained with anti-galectin-3, anti-NLRP3 (Adipogen), and anti-ASC (Adipogen) antibodies. Fluorescenceconjugated secondary antibodies were then used to detect primary antibodies. Cells were mounted on glass slides with Fluoromount-G (Southern Biotech, Birmingham, AL). Immunofluorescent images were acquired with a Zeiss LSM700 confocal microscope (Carl Zeiss, Thornwood, NY) and quantified with Metamorph image analysis software (Molecular Device).

\section{Immunoprecipitation}

J774.1 cells were infected with H5N1 NIBRG-14 virus (multiplicity of infection $=1$ ). At 15 hours after infection, cells were washed with PBS and treated with $8 \mu \mathrm{g} / \mathrm{mL}$ dithiobis succinimidyl propionate cross-linker (SigmaAldrich) for 30 minutes at room temperature. Cross-linking was stopped by the addition of one-tenth volume of $1 \mathrm{~mol} / \mathrm{L}$ Tris- $\mathrm{HCl}$ (pH 7.5), followed by incubation for 15 minutes at room temperature. Next, cells were lyzed in NP-40 buffer $(150 \mathrm{mmol} / \mathrm{L} \mathrm{NaCl}, 25 \mathrm{mmol} / \mathrm{L}$ Tris-HCl, $\mathrm{pH}$ 7.4, $1 \mathrm{mmol} / \mathrm{L}$ EDTA, 0.5\% NP-40, 5\% glycerol) with protease inhibitor cocktail and passed through a 27 -gauge syringe 15 times at $4^{\circ} \mathrm{C}$. Lysates were centrifuged at $550 \times g$ for 8 minutes. Anti-galectin-3 or anti-NLRP3 antibodies (1 mg each) were mixed with protein A/G-Sepharose beads (Merck) overnight at $4{ }^{\circ} \mathrm{C}$. After forming pellets, beads were washed with NP-40 buffer. Total proteins $(100 \mu \mathrm{g})$ were incubated with antibody-conjugated beads overnight at $4{ }^{\circ} \mathrm{C}$. After thorough washes with NP-40 buffer, beads were covered with $50 \mu \mathrm{L}$ of $2 \times$ SDS sample buffer and boiled for 10 minutes. Supernatants with precipitated proteins were collected and separated on 12\% SDS polyacrylamide gels, followed by immunoblotting with anti-galectin-3 and antiNLRP3 antibodies. 


\section{LDH and Protein Concentrations}

Lactate dehydrogenase (LDH) release in BAL collected from infected mice was measured with CytoTox 96 Non-Radioactive Cytotoxicity Assays (Promega, Madison, WI) according to the manufacturer's instructions. Bradford Protein Assays (BioRad) were used to measure total protein concentrations in the BAL samples.

\section{Statistical Analysis}

Statistical differences between experimental groups were analyzed by $t$-tests (GraphPad Prism 6 software; GraphPad Inc., San Diego, CA), with statistical significance established as $P<0.05$. The mouse survival data were analyzed by log-rank (Mantel-Cox) test with GraphPad Prism 6 software (GraphPad Inc.).

\section{Results}

\section{Galectin-3 Is Up-Regulated in Mouse Lungs during H5N1 Infections}

The correlation between galectin-3 expression and $\mathrm{H} 5 \mathrm{~N} 1$ infection was first determined. Lungs collected from H5N1infected and PBS-treated WT mice were homogenized, and galectin-3 levels in lung homogenates were quantified by real-time quantitative PCR and Western blot analysis. Results indicated significant up-regulation of both galectin-3 mRNA (Figure 1A) and protein (Figure 1B) levels after H5N1 infection, suggesting a positive role for galectin-3. Sources of increased galectin-3 in infected lungs were verified. Immunohistochemical staining indicated enhanced galectin-3 staining in H5N1-infected lungs compared with controls treated with PBS (Figure 1C). The observed increase in galectin-3 expression was primarily due to infiltrating cells and to a lesser degree to lung epithelial cells.

\section{Survival of Gal-3KO Mice and WT Mice after H5N1 Infections Is Comparable and the Viral Copies in Lungs Are Also Similar}

Both WT and Gal-3KO mice were used to determine endogenous galectin-3 involvement in $\mathrm{H} 5 \mathrm{~N} 1$ pathogenesis in vivo. A survival rate of approximately $36 \%$ was observed for Gal-3KO mice compared with $0 \%$ for WT mice (Figure 2A); however, no statistically significant difference was found. Although all mice lost body weight after challenges with the $\mathrm{H} 5 \mathrm{~N} 1$ virus, some of the Gal-3KO mice regained weight, starting on day 7 after infection, whereas all WT mice died by day 8 (Figure 2B). Body weight changes were similar between the two genotypes during the first 3 days (Figure 2C).

To address whether galectin-3 affected H5N1 replication in vivo, viral loads in the lungs of both WT and Gal-3KO mice were determined by real-time quantitative PCR between days 1 and 5 after infection. Results indicated the presence of
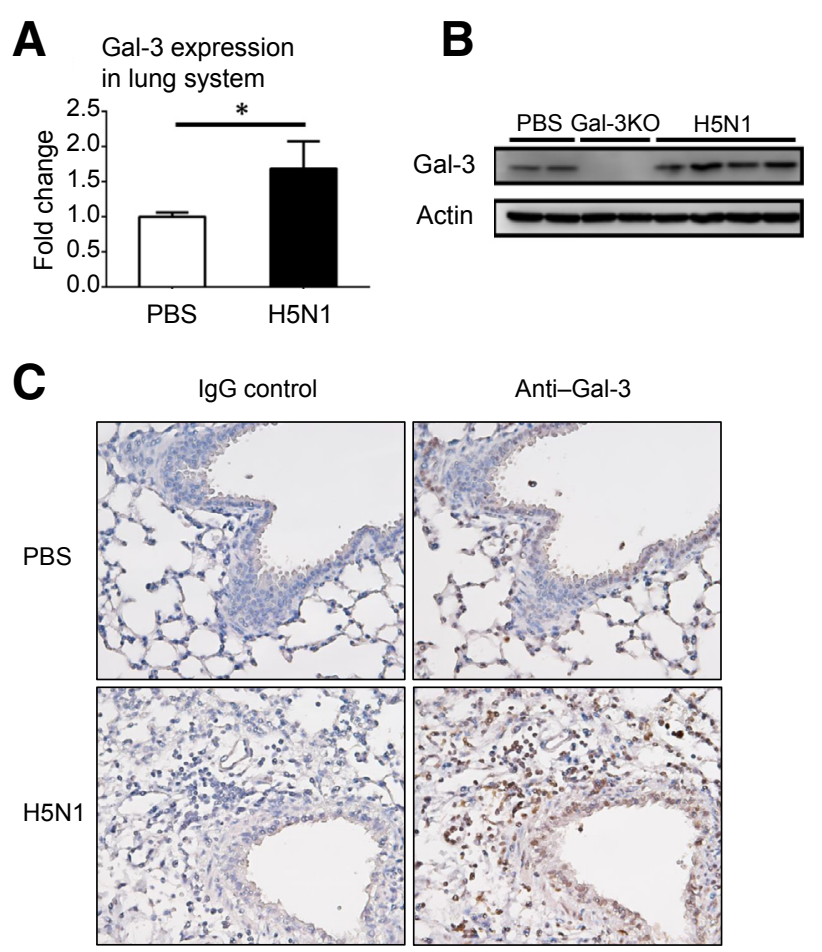

Figure 1 Increased galectin-3 (Gal-3) expression in mouse lungs during H5N1 infections. Wild-type mice were intranasally inoculated with phosphate-buffered saline (PBS) as a control or $10 \times \mathrm{LD}_{50}$ of the H5N1 NIBRG-14 virus. A: Lungs were harvested and homogenized on day 5 after infection. Real-time quantitative PCR was performed to quantify Gal-3 mRNA levels in lung homogenates. B: Gal-3 protein levels in lung homogenates were measured by Western blot analysis. Relative levels of PBStreated versus H5N1-infected tissues were $1.00,1.19$ versus $1.19,1.69$, $1.22,1.35$. C: Results from immunohistochemical staining that used IgG control and anti-Gal-3 antibodies with lung sections from control (PBStreated) and H5N1-infected mice on day 3 after infection. Brown indicates positive reactivity. Data are expressed as means \pm SEM from two independent experiments. $n=6$ per group. ${ }^{*} P<0.05$. Original magnification, $\times 400$. K0, knockout.

similar viral copies in the two genotypes at different times after H5N1 challenges (Figure 2D), indicating the absence of any significant effect from galectin-3 on viral loads.

\section{H5N1-Infected Gal-3K0 Mice Exhibit Reduced Lung Inflammation with Less IL- $1 \beta$ Secretion in Airways Compared with H5N1-Infected WT Mice}

Lung inflammation was next examined in H5N1-infected mice. Hematoxylin and eosin staining indicated lower amounts of inflammatory cells, such as neutrophils, in lung tissue samples from H5N1-infected Gal-3KO mice compared with samples taken from WT mice (Figure 3A). Because excessive inflammation is a known cause of tissue injury, total protein concentrations and LDH levels (two markers of injured lungs) were quantified in BAL fluid collected from both types of mice. Results indicated total protein (Figure 3B) and LDH (Figure 3C) levels in BAL fluids from H5N1-infected Gal-3KO were comparable with WT mice. 

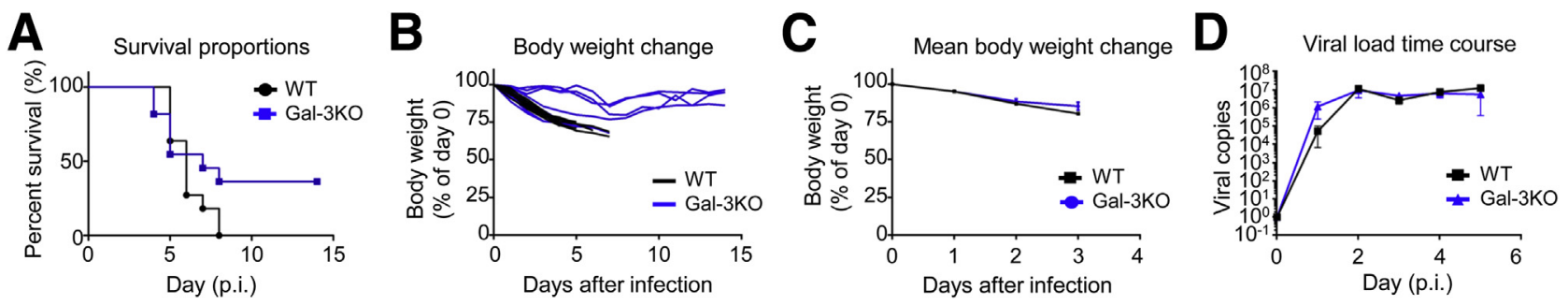

Figure 2 Post-H5N1 infection death, weight loss, and viral burden data. Wild-type (WT) and galectin-3 knockout (Gal-3K0) mice were intranasally inoculated with $10 \times \mathrm{LD}_{50}$ of the H5N1 NIBRG-14 virus or phosphate-buffered saline as a control. A: Mouse survival data for the 14 days after infection (p.i.). B: Body weight change data for individual mice. Weights recorded for 14 days p.i. are expressed as percentages of body weight on day 0 . C: Mean changes in body weights for the first 3 days p.i., when all mice in both groups were still alive. D: Lungs were harvested and homogenized in TRIzol before total RNA extraction between days 1 and 5 p.i. Viral loads were determined at different time points by using real-time quantitative PCR for influenza hemagglutinin proteins. Data are expressed as means \pm SEM. $n=11$ mice per group from 3 independent experiments $(\mathbf{A}-\mathbf{C}) ; n=3$ mice per group from 3 independent experiments (D).

H5N1 has been described as a significant inducer of cytokine response, ${ }^{5}$ and cytokine storms caused by excessive immune responses are known contributors to H5N1 immunopathogenesis. ${ }^{6,7}$ Data from an examination of H5N1induced cytokine production in WT and Gal-3KO mice showed that levels of IL-6 (Figure 3D), TNF- $\alpha$ (Figure 3E), monocyte chemoattractant protein-1 (Figure $3 \mathrm{~F}$ ), and IL- $1 \beta$ levels (Figure 3G) in the lung homogenates of H5N1-infected Gal-3KO mice were comparable with those in WT mice on day 3 after infection. However, the IL-1 $\beta$ levels in BAL fluid from H5N1-infected Gal-3KO mice were significantly lower $(P<0.05)$ compared with those from H5N1-infected WT mice (Figure $3 \mathrm{H}$ ). This indicated that galectin-3 up-regulated H5N1-induced IL-1 $\beta$ production. Combined, these results suggested that galectin-3 may enhance H5N1 effects by regulating IL-1 $\beta$ production and inflammatory responses.

\section{Gal-3KO BMMs Secrete Less IL-1 $\beta$ than WT BMMs in} Response to H5N1 Infection while Expressing Similar NLRP3 Inflammasome Component Levels

In addition to serving as H5N1 infection targets in lung tissue, macrophages are key producers of proinflammatory cytokines such as IL-1 $\beta$ and TNF- $\alpha .{ }^{8,33}$ Therefore, BMMs were used to study the role of galectin- 3 in IL- $1 \beta$ production after $\mathrm{H} 5 \mathrm{~N} 1$ challenges in vitro. Results indicated that BMMs from Gal-3KO mice produced lower quantities of IL-1 $\beta 18$ hours after infection $(P<0.05)$ compared with BMMs from WT mice (Figure $4 \mathrm{~A}$ ). Influenza viruses are known inducers of IL-1 $\beta$ production by NLRP3 inflammasome activation. ${ }^{13,14}$ This activation required two signals: a toll-like receptor-NF- $\mathrm{KB}$ signaling pathway and NLRP3 inflammasome assembly. ${ }^{17}$ To verify the role of galectin-3 in NLRP3 inflammasome activation, pro-IL-1 $\beta$ and NLRP3 mRNA levels were examined in H5N1-infected WT and Gal-3KO BMMs. Similar levels of the two mRNAs (Figure 4B) were found, indicating a lack of galectin-3 involvement in $\mathrm{H} 5 \mathrm{~N} 1$ infection-induced NF- $\mathrm{KB}$ pathways. In contrast, Western blot data showed that at 15 hours after infection, Gal-3KO BMMs secreted smaller amounts of mature IL-1 $\beta$ into supernatant compared with WT BMMs (Figure 4C). Similarities were also found in NLRP3 inflammasome component expression at the protein level (ie, NLRP3, ASC, pro-IL-1 $\beta$, and pro-caspase-1) (Figure 4C). These results suggested that the effects of galectin- 3 on IL- $1 \beta$ production in macrophages during $\mathrm{H} 5 \mathrm{~N} 1$ infections were not associated with regulation of the toll-like receptor-NF- $\kappa \mathrm{B}$ signaling pathway.

\section{Galectin-3 Promotes ASC Pyroptosome Formation and NLRP3 Inflammasome Assembly and Is Associated with NLRP3 and ASC Speck}

NLRP3 activation has been shown to induce the formation of ASC oligomers or dimers (ASC pyroptosomes), resulting in the recruitment and activation of caspase- $1 .{ }^{34}$ Accordingly, ASC oligomerization served as an indicator of inflammasome activation. To determine whether galectin-3 regulated ASC pyroptosome formation during $\mathrm{H} 5 \mathrm{~N} 1$ infections, WT and Gal-3KO BMMs were used to perform ASC pyroptosome cross-linking assays. Results indicated lower levels of ASC oligomers in Gal-3KO BMMs compared with WT BMMs at 24 hours after infection (Figure 5A), suggesting that galectin-3 enhances NLRP3 inflammasome assembly and ASC oligomerization. To verify galectin-3 regulation of NLRP3 inflammasome assembly, NLRP3 inflammasome complex was reconstituted in HEK293T cells by transfecting specific inflammasome components, resulting in the bypassing of the NF- $\kappa \mathrm{B}$ pathway and in autonomous IL-1 $\beta$ secretion. Immunoblotting data showed that galectin-3 co-transfection dosedependently enhanced the secretion of mature IL-1 $\beta 48$ hours after transfection (Figure 5B). This indicted that galectin-3 promoted NLRP3 inflammasome assembly and activation in addition to $\mathrm{IL}-1 \beta$ processing and secretion. Combined, these results suggested that during H5N1 infections galectin- 3 regulated IL- $1 \beta$ production by promoting NLRP3 inflammasome assembly and ASC pyroptosome formation. 
A
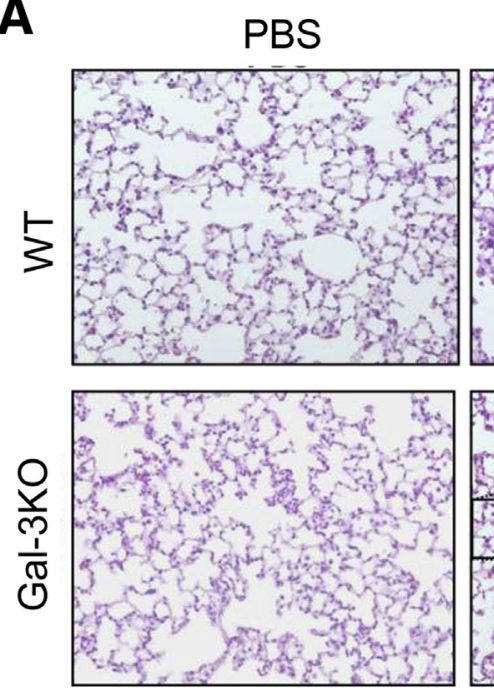

B

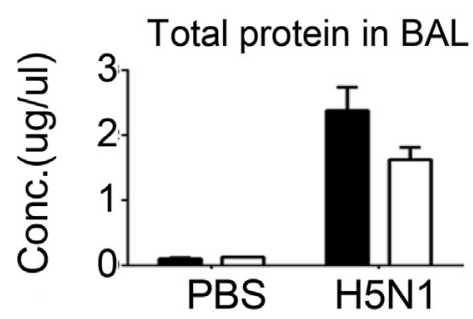

D



$\mathbf{F}$

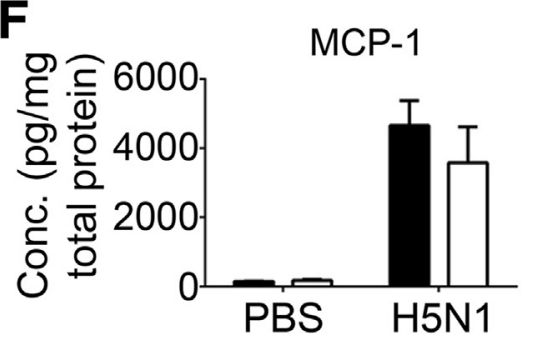

H5N1


C

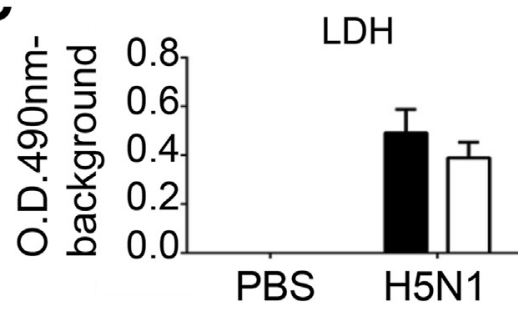

E



Figure 3 Galectin-3 knockout (Gal-3KO) mice exhibit reduced lung inflammation, neutrophil infiltration, and lung injuries, and they express lower levels of IL- $1 \beta$ compared with wild-type (WT) mice during H5N1 infections. WT and Gal-3K0 mice were intranasally inoculated with $10 \times \mathrm{LD}_{50}$ of the H5N1 NIBRG-14 virus or phosphatebuffered saline (PBS) as control and sacrificed on day 3 after infection. A: Lung tissues were fixed, paraffin embedded, and sectioned for hematoxylin and eosin staining. Insets: Higher magnification of the boxed areas in main images. Arrows indicate neutrophils. B and C: Levels of total protein $(P=0.06 ; B)$ and lactate dehydrogenase $(\mathrm{LDH})(P=0.31 ; \mathrm{C})$ in bronchoalveolar lavage (BAL) fluid were measured by using Bradford protein and LDH cytotoxicity assays, respectively. D-G: Concentrations of IL-6 (D), tumor necrosis factor- $\alpha$ (TNF- $\alpha ; E)$, monocyte chemoattractant protein-1 (MCP-1; F), and IL-1 $\beta$ $(P=0.09 ; \mathbf{G})$ in the supernatants of lung homogenates, as measured by enzymelinked immunosorbent assays (ELISAs) and normalized to total protein concentrations. No statistically significant differences were noted for B through G. H: IL-1 $\beta$ concentrations in BAL fluid were measured by ELISAs. Black bars, WT; white bars, Gal-3KQ (B-H). Data are expressed as means \pm SEM. $n=4$ mice per group from 3 independent experiments. ${ }^{*} P<0.05$. Original magnification: $\times 200$ (A, main images); $\times 600$ (A, insets). Conc, concentration.


To determine whether galectin-3 regulated NLRP3 inflammasome assembly through direct interaction with inflammasome components, immunofluorescence assays were used to examine galectin-3 and NLRP3 inflammasome localization in transfected HEK293T cells. The presence of galectin-3 enhanced ASC speck formation and ASCNLRP3 association (Figure 5, C and D). In addition, associations of galectin-3 were observed with both ASC speck and NLRP3 inflammasome complex (Figure 5). Co-immunoprecipitation assays were performed to determine the association between galectin-3 and NLRP3 during $\mathrm{H} 5 \mathrm{~N} 1$ infections. NLRP3 was pulled down when galectin-3 was immunoprecipitated in an H5N1-infected mouse macrophage J774A.1 cell line (Figure 5E), suggesting galectin-3 interaction with NLRP3 during H5N1 infections. 

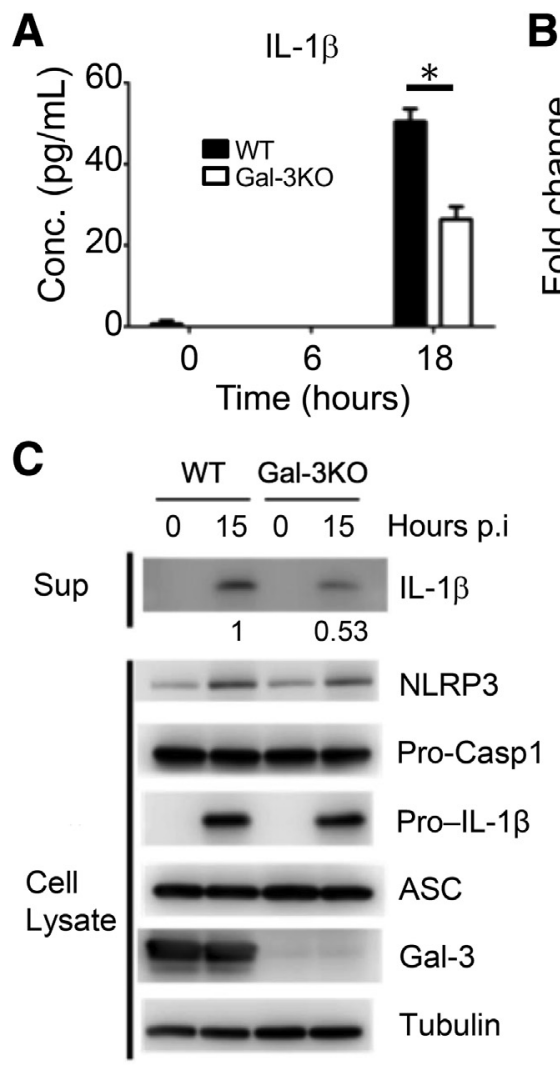


\begin{abstract}
Figure 4 Galectin-3 knockout (Gal-3KO) bone marrow-derived macrophages (BMMs) produce lower levels of IL-1 $\beta$ compared with wild-type (WT) BMMs in response to H5N1 infections, but nucleotide oligomerization domain-like receptor protein 3 (NLRP3) component expression is similar. BMMs from WT and Gal-3K0 mice were infected with H5N1. A: IL-1 $\beta$ levels in cell-free supernatants were measured by enzyme-linked immunosorbent assays. B: Cells were lyzed for RNA extraction at different time points; pro-IL-1 $\beta$ and NLRP3 mRNA levels were measured by real-time quantitative PCR. C: Mature IL-1 $\beta$ in cell-free supernatant (Sup) was precipitated with trichloroacetic acid and measured by Western blot analysis. Protein levels of NLRP3, pro-caspase-1 (pro-Casp1), pro-IL-1 $\beta$, apoptosis-associated speck-like protein containing a caspase-associated recruitment domain (ASC), and galectin- 3 in cell lysates were measured by Western blot analysis; $\alpha$-tubulin was used as an internal control. IL-1 $\beta$ level in supernatant from Gal-3KO was $53 \%$ that from WT. Data are expressed as means \pm SEM. $n=3$ samples from 2 independent experiments (A and $\mathbf{B}) ; n=3$ separate experiments (C). ${ }^{*} P<0.05$. Conc., concentration; p.i., after infection.
\end{abstract}

\section{Discussion}

Galectin-3 is involved in a number of infectious and lungcentered diseases. ${ }^{29-31,35}$ However, its role in pulmonary influenza virus-induced host response has not been fully evaluated previously. We found evidence to indicate that endogenous galectin-3 enhances H5N1-induced inflammation and IL- $1 \beta$ production by functioning intracellularly and promoting NLRP3 inflammasome complex formation.

After treatment with large doses $\left(10 \mathrm{LD}_{50}\right)$ of $\mathrm{H} 5 \mathrm{~N} 1$ NIBRG-14, significantly lower IL-1 $\beta$ levels were observed in the airways of Gal-3KO mice and moderate lung inflammation 3 days after infection (Figure 3). No statistically significant differences between Gal-3KO and WT mice were noted in terms of body weight loss, death, or viral loads (Figure 2). The observations for Gal-3KO mice challenged with large doses of $\mathrm{H} 5 \mathrm{~N} 1$ with regard to lung inflammation were similar to previously reported outcomes for IL-1R-deficient mice, ${ }^{10,36}$ suggesting that the effects of galectin-3 on IAV-induced inflammation may be primarily due to IL-1 $\beta$ production regulation. However, unlike that resulting from IL-1R deficiency, survival of Gal-3KO mice was comparable with WT mice. Thus, galectin-3 may affect certain pathologic responses to IAV infections by other mechanisms besides IL-1 $\beta$ production control.

Several efforts have been made to use lung macrophages or BMMs to evaluate avian IAV-induced immune responses and cytokine production. ${ }^{37,38}$ Their data indicate that lung-based macrophage populations are heterogeneous, consisting of alveolar macrophages within airways plus interstitial and exudate-derived macrophages. Steady-state alveolar macrophages have been described as alternatively activated, with an M2-like anti-inflammatory phenotype thought to maintain lung homoeostasis. ${ }^{37-41}$ We surmised that classically activated M1-like BMMs induced by granulocyte-macrophage colony-stimulating factor with a proinflammatory phenotype might be more suitable for identifying the role of galectin-3 H5N1-induced proinflammatory response, especially inflammasome activation.

Researchers have described the contributions of stronger and prolonged viral replication, differences in cell tropism, broader tissue dissemination of viruses, and aberrant host immune responses to severe human $\mathrm{H} 5 \mathrm{~N} 1$ pathogenesis. ${ }^{4,6}$ Further, lung pathology and progressive respiratory failure are considered primary factors that contribute to human H5N1-related death. ${ }^{4}$ Although researchers have shown that innate immune responses are rapidly induced to control viral replication, ${ }^{42}$ they have also demonstrated that the infiltration of excessive neutrophils into airways are associated with immunopathology during infections with highly virulent influenza. ${ }^{43}$ Neutrophils have been reported as key contributors to acute respiratory distress syndrome. ${ }^{44} \mathrm{Gal}-3 \mathrm{KO}$ mice exhibited less neutrophil infiltration and pulmonary inflammation compared with WT mice (Figure 3A), suggesting that galectin-3 promotes $\mathrm{H} 5 \mathrm{~N} 1$ disease-associated inflammation. 
For the past 20 years, H5N1 influenza has evolved extensively and been classified according to their HA gene sequences. Evidence indicates their divergence into at least 40 clades,${ }^{45}$ with only four currently circulating globally (clades 2.2.1.2; 2.3.2.1a; 2.3.2.1c; and 2.3.4.4.6). ${ }^{45-47}$ For this study H5N1 vaccine strain A/Vietnam/1203/04 was used, which belongs to clade 1 . Because a single H5N1 vaccine strain was used to evaluate the effects of galectin-3

A

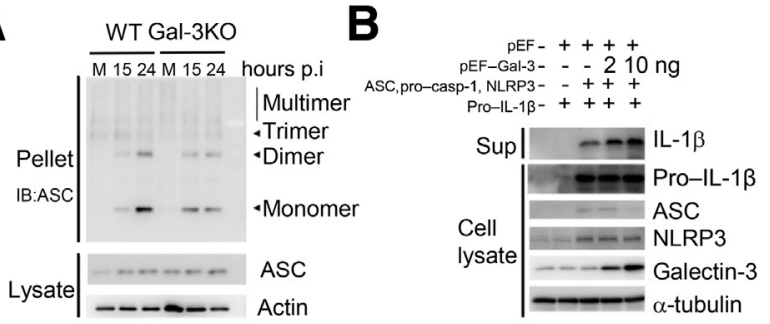

C
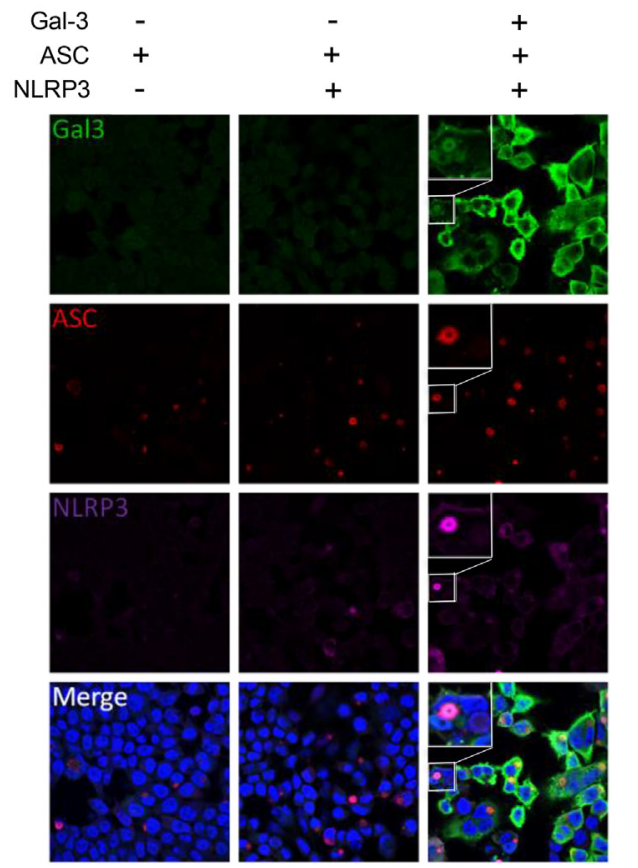

D
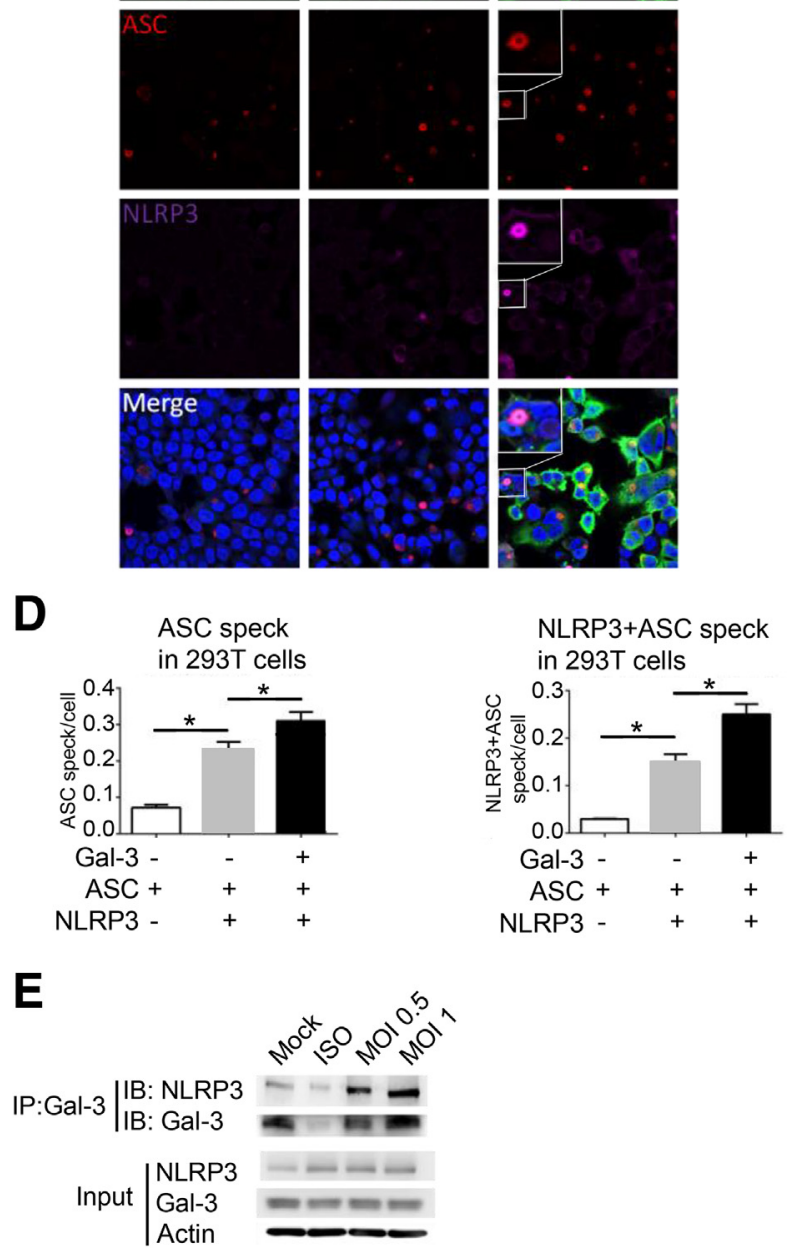

on $\mathrm{H} 5 \mathrm{~N} 1$ infection, additional studies are required to determine whether the promoting effect of the galectin-3 is generalizable to all H5N1 clades.

Hypercytokinemia is a major feature of human H5N1 immunopathogenesis. ${ }^{5-7}$ Severe lung inflammation can lead to more obvious clinical symptoms and potentially lethal lung injuries. ${ }^{48,49}$ Data from the comparison of cytokine expression in lung homogenates from H5N1-infected mice indicate significantly lower IL- $1 \beta$ levels in BAL fluid collected from Gal-3KO mice compared with WT mice (Figure $3 \mathrm{H}$ ). These findings suggest that galectin-3 enhances proinflammatory environments during $\mathrm{H} 5 \mathrm{~N} 1$ infection. Note that the difference in IL- $1 \beta$ levels in lung homogenates from infected WT and Gal-3KO mice were not statistically significant (Figure 3G). Because ELISAs detect both proand mature forms of IL-1 $\beta$ in lung homogenates, IL- $1 \beta$ secretions in BAL fluid may be more representative of mature IL-1 $\beta$ levels.

Several efforts have been made to clarify the role of endogenous galectin-3 in infection-induced inflammatory response during microbial infections. One research team found that Gal3KO mice subjected to pulmonary infections that involved Francisella novicida (a Gram-negative pathogen that causes severe sepsis) exhibited reductions in neutrophil infiltration, vascular injury, and inflammatory cytokine production, including IL-1 $\beta$, TNF- $\alpha$, and interferon- $\gamma .{ }^{50}$ These mice experienced improved survival with bacterial burdens similar to a group of WT mice, suggesting that galectin-3 plays a pathogenic role in sepsis development by increasing inflammatory response during $F$. novicida infections. ${ }^{50}$ These findings are consistent with our conclusion that galectin-3 enhances H5N1 pathogenic effects by promoting IL-1 $\beta$ production and pulmonary inflammation, with little change in viral load. However, in a study that involved Rhodococcus equi (an intracellular macrophage bacterium), Gal-3KO mice developed delayed but

Figure 5 Galectin-3 (Gal-3) enhances apoptosis-associated speck-like protein containing a caspase-associated recruitment domain (ASC) oligomerization and nucleotide oligomerization domain-like receptor protein (NLRP3) inflammasome activation, and associates with both NLRP3 and ASC speck. A: Wild-type (WT) and Gal-3 knockout (Gal-3KO) bone marrow-derived macrophages were infected with $\mathrm{H} 5 \mathrm{~N} 1$ at the multiplicity of infection (MOI) of 1 . ASC oligomers in cells were cross-linked with dextran sulfate sodium and measured by Western blot analysis. B: HEK293T cells were transfected with plasmids by using Effectene as indicated. Supernatants (Sup) and cell extracts were collected after 48 hours. Levels of IL-1 $\beta$ in supernatants and levels of pro-IL$1 \beta$, NLRP3, ASC, and Gal-3 in cell extracts were measured by Western blot analysis. C: Immunofluorescence microscopy images of Gal-3, NLRP3, and ASC in transfected HEK293T cells 48 hours after transfection (p.i.). Insets: Higher magnification of the boxed areas in main images. D: Quantitative results for $\mathbf{C}$ ASC specks and ASC-NLRP3 associations per cell. E: J774.1 cells were infected with H5N1 (MOI $=0.5$ or 1 ). Cells were cross-linked with dithiobissuccinimidylpropionate and harvested for immunoprecipitation (IP) assays 15 hours p.i. Gal-3 was immune-precipitated with anti-Gal-3 antibodies. Co-precipitated NLRP3 was detected by immunoblot (IB) analysis. Rabbit IgG was used as an isotype control (ISO). All results are representative of independently conducted experiments. Data are expressed as means \pm SEM. $n=2$ independent experiments. ${ }^{*} P<0.05$. Original magnification: $\times 400$ (C, main images); $\times 920$ (C, insets). Casp1, caspase-1. 
stronger inflammatory reactions with higher levels of IL-12, interferon- $\gamma$, and IL-1 $\beta .^{51}$ These findings suggest that galectin-3 alters mouse sensitivity to $R$. equi by reducing IL- $1 \beta$ production from macrophages, ${ }^{51}$ which conflicts with our finding that galectin- 3 promotes $\mathrm{IL}-1 \beta$ production. These contradictory results may be due to the inducement of different IL$1 \beta$ production and/or galectin-3-associated immune system regulation mechanisms. It may be that the roles of galectin-3 in infection-induced immune responses differ depending on the pathogens and organs involved.

The cell-based data indicate an association between galectin-3 and NLRP3 (Figure 5D) that affects ASC oligomerization (Figure $5 \mathrm{~A}$ ) and IL- $1 \beta$ production. No effect was observed on NF- $\mathrm{BB}$ signaling, as indicated by similar levels of pro-IL-1 $\beta$ and NLRP3 transcripts and proteins in macrophages collected from H5N1-infected WT and Gal-3KO mice (Figure 4, B and C). In the NLRP3 inflammasome reconstitution system, galectin-3 promoted NLRP3-ASC inflammasome assembly (Figure 5, C and D). A possible explanation is that binding with galectin-3 triggers conformational changes in NLRP3, which in turn activates NLRP3 and induces ASC oligomerization. In summary, our data support the idea that galectin-3 serves as a bridge for NLRP3-ASC interaction and complex formation.

The conclusion that galectin-3 promotes NLRP3-ASC inflammasome assembly and IL-1 $\beta$ processing, suggests that the positive effect of galectin-3 on NLRP3 inflammasome complex formation may not be restricted to $\mathrm{H} 5 \mathrm{~N} 1$ infections. Pro-IL-1 $\beta$ levels in the lysates of cells transfected with pro-IL-1 $\beta$ plasmids were much lower than in cells transfected with other inflammasome components (Figure 5B). According to this observation, it is likely that the presence and assembly of NLRP3 inflammasome components may stabilize pro-IL- $1 \beta$ in cells. The presence of a basal amount of ASC speck was noted in ASC-expressing 293T cells (Figure 5C), perhaps because of protein overproduction.

Other researchers have described the role of galectin-3 in NLRP3-mediated IL-1 $\beta$ production. In studies that involved dextran sulfate sodium-induced colitis and primary biliary cholangitis, Gal-3KO mice exhibited reduced disease severity, decreases in the production of IL-1 $\beta$ and other proinflammatory cytokines, and lower NLRP3 expression compared with WT mice. ${ }^{52,53}$ These studies suggest a positive role for galectin-3 in the NF- $\kappa B$ pathway, which contrasts with our finding that galectin-3 specifically acts on NLRP3 inflammasome assembly. It may be that galectin-3 regulatory mechanisms linked with IL-1 $\beta$ production differ, depending on the disease model being used. During IAV infections, various virus components sensed by cells are known to trigger different downstream inflammatory signals-examples include the IAV proteins M2, NS1, and PB1 and virus RNA, all of which have been described as triggering or inhibiting NLRP3 inflammasome activation. ${ }^{13,54-56}$ Researchers may be interested in investigating the potential involvement of galectin-3 in these processes.

\section{Conclusions}

Our results indicate that after H5N1 infection i) endogenous galectin-3 enhances IL-1 $\beta$ production and NLRP3 inflammasome assembly but not NF- $\mathrm{BB}$ pathways in macrophages and ii) galectin-3 and NLRP3 interaction increases NLRP3ASC inflammasome assembly and ASC oligomerization. From our finding that endogenous galectin-3 plays a key role in H5N1-induced inflammation, targeting galectin-3 may hold therapeutic potential for H5N1-induced pneumonia and lung injuries by attenuating inflammatory responses. We also accumulated additional evidence about the effects of galectin-3 in H5N1 infections that involve potent cytokine storms and lung inflammation. ${ }^{5,6}$ Further study is required to determine whether the role of galectin-3 is restricted to $\mathrm{H} 5 \mathrm{~N} 1$ or is common among IAV strains.

\section{References}

1. Korteweg C, Gu J: Pathology, molecular biology, and pathogenesis of avian influenza A (H5N1) infection in humans. Am J Pathol 2008, 172:1155-1170

2. Skeik N, Jabr FI: Influenza viruses and the evolution of avian influenza virus H5N1. Int J Infect Dis 2008, 12:233-238

3. Thanh TT, van Doorn HR, de Jong MD: Human H5N1 influenza: current insight into pathogenesis. Int J Biochem Cell Biol 2008, 40: 2671-2674

4. Peiris JS, Cheung CY, Leung CY, Nicholls JM: Innate immune responses to influenza A H5N1: friend or foe? Trends Immunol 2009, 30:574-584

5. de Jong MD, Simmons CP, Thanh TT, Hien VM, Smith GJ, Chau TN, Hoang DM, Chau NV, Khanh TH, Dong VC, Qui PT, Cam BV, Ha do Q, Guan Y, Peiris JS, Chinh NT, Hien TT, Farrar J: Fatal outcome of human influenza A (H5N1) is associated with high viral load and hypercytokinemia. Nat Med 2006, 12:1203-1207

6. Beigel JH, Farrar J, Han AM, Hayden FG, Hyer R, de Jong MD, Lochindarat S, Nguyen TK, Nguyen TH, Tran TH, Nicoll A, Touch S, Yuen KY; Writing Committee of the World Health Organization (WHO) Consultation on Human Influenza A/H5: Avian influenza A (H5N1) infection in humans. N Engl J Med 2005, 353 : $1374-1385$

7. Damjanovic D, Small CL, Jeyanathan M, McCormick S, Xing Z Immunopathology in influenza virus infection: uncoupling the friend from foe. Clin Immunol 2012, 144:57-69

8. Snelgrove RJ, Goulding J, Didierlaurent AM, Lyonga D, Vekaria S, Edwards L, Gwyer E, Sedgwick JD, Barclay AN, Hussell T: A critical function for CD200 in lung immune homeostasis and the severity of influenza infection. Nat Immunol 2008, 9:1074-1083

9. Lin KL, Suzuki Y, Nakano H, Ramsburg E, Gunn MD: CCR2+ monocyte-derived dendritic cells and exudate macrophages produce influenza-induced pulmonary immune pathology and mortality. J Immunol 2008, 180:2562-2572

10. Schmitz N, Kurrer M, Bachmann MF, Kopf M: Interleukin-1 is responsible for acute lung immunopathology but increases survival of respiratory influenza virus infection. J Virol 2005, 79:6441-6448

11. Sims JE, Smith DE: The IL-1 family: regulators of immunity. Nat Rev Immunol 2010, 10:89-102

12. Dinarello CA: Biologic basis for interleukin-1 in disease. Blood 1996, 87:2095-2147

13. Allen IC, Scull MA, Moore CB, Holl EK, McElvania-TeKippe E, Taxman DJ, Guthrie EH, Pickles RJ, Ting JP: The NLRP3 
inflammasome mediates in vivo innate immunity to influenza A virus through recognition of viral RNA. Immunity 2009, 30:556-565

14. Thomas PG, Dash P, Aldridge JR Jr, Ellebedy AH, Reynolds C, Funk AJ, Martin WJ, Lamkanfi M, Webby RJ, Boyd KL, Doherty PC, Kanneganti TD: The intracellular sensor NLRP3 mediates key innate and healing responses to influenza $\mathrm{A}$ virus via the regulation of caspase-1. Immunity 2009, 30:566-575

15. Ichinohe T, Lee HK, Ogura Y, Flavell R, Iwasaki A: Inflammasome recognition of influenza virus is essential for adaptive immune responses. J Exp Med 2009, 206:79-87

16. Bauernfeind FG, Horvath G, Stutz A, Alnemri ES, MacDonald K, Speert D, Fernandes-Alnemri T, Wu J, Monks BG, Fitzgerald KA, Hornung V, Latz E: Cutting edge: NF-kappaB activating pattern recognition and cytokine receptors license NLRP3 inflammasome activation by regulating NLRP3 expression. J Immunol 2009, 183: 787-791

17. Pang IK, Iwasaki A: Inflammasomes as mediators of immunity against influenza virus. Trends Immunol 2011, 32:34-41

18. Schroder K, Tschopp J: The inflammasomes. Cell 2010, 140: 821-832

19. Barondes SH, Cooper DN, Gitt MA, Leffler H: Galectins. Structure and function of a large family of animal lectins. J Biol Chem 1994, 269:20807-20810

20. Ahmad N, Gabius HJ, Andre S, Kaltner H, Sabesan S, Roy R, Liu B, Macaluso F, Brewer CF: Galectin-3 precipitates as a pentamer with synthetic multivalent carbohydrates and forms heterogeneous cross-linked complexes. J Biol Chem 2004, 279: 10841-10847

21. Liu FT, Rabinovich GA: Galectins: regulators of acute and chronic inflammation. Ann N Y Acad Sci 2010, 1183:158-182

22. Dumic J, Dabelic S, Flogel M: Galectin-3: an open-ended story. Biochim Biophys Acta 2006, 1760:616-635

23. Liu FT, Patterson RJ, Wang JL: Intracellular functions of galectins. Biochim Biophys Acta 2002, 1572:263-273

24. Chen HY, Liu FT, Yang RY: Roles of galectin-3 in immune responses. Arch Immunol Ther Exp (Warsz) 2005, 53:497-504

25. Vasta GR: Roles of galectins in infection. Nat Rev Microbiol 2009, 7 : 424-438

26. Norling LV, Perretti M, Cooper D: Endogenous galectins and the control of the host inflammatory response. J Endocrinol 2009, 201: 169-184

27. Hsu DK, Yang RY, Pan Z, Yu L, Salomon DR, Fung-Leung WP, Liu FT: Targeted disruption of the galectin-3 gene results in attenuated peritoneal inflammatory responses. Am J Pathol 2000, 156: $1073-1083$

28. Colnot C, Ripoche MA, Milon G, Montagutelli X, Crocker PR, Poirier F: Maintenance of granulocyte numbers during acute peritonitis is defective in galectin-3-null mutant mice. Immunology 1998, 94:290-296

29. Nieminen J, St-Pierre C, Bhaumik P, Poirier F, Sato S: Role of galectin-3 in leukocyte recruitment in a murine model of lung infection by Streptococcus pneumoniae. J Immunol 2008, 180: $2466-2473$

30. Pilette C, Colinet B, Kiss R, Andre S, Kaltner H, Gabius HJ, Delos M, Vaerman JP, Decramer M, Sibille Y: Increased galectin-3 expression and intra-epithelial neutrophils in small airways in severe COPD. Eur Respir J 2007, 29:914-922

31. Zuberi RI, Hsu DK, Kalayci O, Chen HY, Sheldon HK, Yu L, Apgar JR, Kawakami T, Lilly CM, Liu FT: Critical role for galectin-3 in airway inflammation and bronchial hyperresponsiveness in a murine model of asthma. Am J Pathol 2004, $165: 2045-2053$

32. Wood JM, Robertson JS: From lethal virus to life-saving vaccine: developing inactivated vaccines for pandemic influenza. Nat Rev Microbiol 2004, 2:842-847

33. Hofmann P, Sprenger H, Kaufmann A, Bender A, Hasse C, Nain M, Gemsa D: Susceptibility of mononuclear phagocytes to influenza A virus infection and possible role in the antiviral response. J Leukoc Biol 1997, 61:408-414

34. Fernandes-Alnemri T, Alnemri ES: Assembly, purification, and assay of the activity of the ASC pyroptosome. Methods Enzymol 2008, 442:251-270

35. Sato S, Ouellet N, Pelletier I, Simard M, Rancourt A, Bergeron MG: Role of galectin-3 as an adhesion molecule for neutrophil extravasation during streptococcal pneumonia. J Immunol 2002, 168: $1813-1822$

36. Szretter KJ, Gangappa S, Lu X, Smith C, Shieh WJ, Zaki SR, Sambhara S, Tumpey TM, Katz JM: Role of host cytokine responses in the pathogenesis of avian H5N1 influenza viruses in mice. J Virol 2007, 81:2736-2744

37. Ettensohn DB, Roberts NJ Jr: Influenza virus infection of human alveolar and blood-derived macrophages: differences in accessory cell function and interferon production. J Infect Dis 1984, 149: 942-949

38. Dutia MB, Nicol MQ: The role of macrophages in influenza A virus infection. Future Virol 2014, 9:847-862

39. Campbell GM, Nicol MQ, Dransfield I, Shaw DJ, Nash AA, Dutia BM: Susceptibility of bone marrow-derived macrophages to influenza virus infection is dependent on macrophage phenotype. J Gen Virol 2015, 96:2951-2960

40. Ettensohn DB, Frampton MW, Nichols JE, Roberts NJ Jr: Human alveolar macrophages may not be susceptible to direct infection by a human influenza virus. J Infect Dis 2016, 214:1658-1665

41. Lee SM, Dutry I, Peiris JS: Editorial: macrophage heterogeneity and responses to influenza virus infection. J Leukoc Biol 2012, 92:1-4

42. Kreijtz JH, Fouchier RA, Rimmelzwaan GF: Immune responses to influenza virus infection. Virus Res 2011, 162:19-30

43. Tumpey TM, Garcia-Sastre A, Taubenberger JK, Palese P, Swayne DE, Pantin-Jackwood MJ, Schultz-Cherry S, Solorzano A, Van Rooijen N, Katz JM, Basler CF: Pathogenicity of influenza viruses with genes from the 1918 pandemic virus: functional roles of alveolar macrophages and neutrophils in limiting virus replication and mortality in mice. J Virol 2005, 79:14933-14944

44. Williams AE, Chambers RC: The mercurial nature of neutrophils: still an enigma in ARDS? Am J Physiol Lung Cell Mol Physiol 2014, 306:L217-L230

45. Marinova-Petkova A, Franks J, Tenzin S, Dahal N, Dukpa K, Dorjee J, Feeroz MM, Rehg JE, Barman S, Krauss S, McKenzie P, Webby RJ, Webster RG: Highly pathogenic reassortant avian influenza $\mathrm{A}(\mathrm{H} 5 \mathrm{~N} 1)$ virus clade 2.3.2.1a in poultry, Bhutan. Emerg Infect Dis 2016, 22:2137-2141

46. Bi Y, Chen J, Zhang Z, Li M, Cai T, Sharshov K, Susloparov I, Shestopalov A, Wong G, He Y, Xing Z, Sun J, Liu D, Liu Y, Liu L, Liu W, Lei F, Shi W, Gao GF: Highly pathogenic avian influenza H5N1 Clade 2.3.2.1c virus in migratory birds, 2014-2015. Virol Sin 2016, 31:300-305

47. Pearce MB, Pappas C, Gustin KM, Davis CT, Pantin-Jackwood MJ, Swayne DE, Maines TR, Belser JA, Tumpey TM: Enhanced virulence of clade 2.3.2.1 highly pathogenic avian influenza A H5N1 viruses in ferrets. Virology 2017, 502:114-122

48. La Gruta NL, Kedzierska K, Stambas J, Doherty PC: A question of self-preservation: immunopathology in influenza virus infection. Immunol Cell Biol 2007, 85:85-92

49. Shaw MW, Arden NH, Maassab HF: New aspects of influenza viruses. Clin Microbiol Rev 1992, 5:74-92

50. Mishra BB, Li Q, Steichen AL, Binstock BJ, Metzger DW, Teale JM, Sharma J: Galectin-3 functions as an alarmin: pathogenic role for sepsis development in murine respiratory tularemia. PLoS One 2013, 8:e59616

51. Ferraz LC, Bernardes ES, Oliveira AF, Ruas LP, Fermino ML, Soares SG, Loyola AM, Oliver C, Jamur MC, Hsu DK, Liu FT, Chammas R, Roque-Barreira MC: Lack of galectin-3 alters the balance of innate immune cytokines and confers resistance to Rhodococcus equi infection. Eur J Immunol 2008, 38:2762-2775 
52. Simovic Markovic B, Nikolic A, Gazdic M, Bojic S, Vucicevic L, Kosic M, Mitrovic S, Milosavljevic M, Besra G, Trajkovic V, Arsenijevic N, Lukic ML, Volarevic V: Galectin-3 plays an important pro-inflammatory role in the induction phase of acute colitis by promoting activation of NLRP3 inflammasome and production of IL1beta in macrophages. J Crohns Colitis 2016, 10:593-606

53. Tian J, Yang G, Chen HY, Hsu DK, Tomilov A, Olson KA, Dehnad A, Fish SR, Cortopassi G, Zhao B, Liu FT, Gershwin ME, Torok NJ, Jiang JX: Galectin-3 regulates inflammasome activation in cholestatic liver injury. FASEB J 2016, 30:4202-4213
54. Ichinohe T, Pang IK, Iwasaki A: Influenza virus activates inflammasomes via its intracellular M2 ion channel. Nat Immunol 2010, 11:404-410

55. Cheong WC, Kang HR, Yoon H, Kang SJ, Ting JP, Song MJ: Influenza A virus NS1 protein inhibits the NLRP3 inflammasome. PLoS One 2015, 10:e126456

56. McAuley JL, Tate MD, MacKenzie-Kludas CJ, Pinar A, Zeng W, Stutz A, Latz E, Brown LE, Mansell A: Activation of the NLRP3 inflammasome by IAV virulence protein PB1-F2 contributes to severe pathophysiology and disease. PLoS Pathog 2013, 9: e1003392 